\title{
Direct Determination of Aluminium Concentration in Human Blood through Laser Photoionization Spectroscopy
}

\author{
G. I. BEKOV, V. S. LETOKHOV and V. N. RADAYEV
}

Institute of Spectroscopy, USSR Academy of Sciences, 142092, Moscow Region, Troitsk, USSR

\section{(Received 14 September 1982)}

The results of aluminium content measurements in human blood by the method of laser stepwise photoionization of atoms in combination with vacuum thermal atomization of organic substance are presented. The analytical procedure was as follows. Dry blood residium obtained after drying of $40 \mu \mathrm{l}$ natural blood was stepwise heated in vacuum to $1800^{\circ} \mathrm{C}$. The aluminium atoms produced in an atomic-molecular beam were excited to a Rydberg state in two steps by laser radiation and then efficiently ionized by electric field pulse. The resulting ions were detected with an electron multiplier. The calibration curve obtained for aqueous solutions of $\mathrm{AlCl}_{3}$ was used for quantitative determination of aluminium content. The measured value of aluminium concentration in human blood came to $(230 \pm 50) \mathrm{ng} / \mathrm{ml}$.

The determination of trace amounts of metals in biological systems is one of the urgent analytical problems today in biology and medicine. Such analyses are important in order to determine normal concentrations of rnetal traces in an organism as well as correlating their variations from the normal value with different functional disorders and diseases.

With the development of the analytical technique the sensitivity, accuracy and reliability of measurement have substantially increased. Ideas about the role of metals in an organism have therewith changed. Such elements as iron, cobalt and nickel which were believed to be harmless become a deadly poison if their concentration increases 
sharply. The presence of Ni: $1.1 \div 4.6 \mathrm{ng} / \mathrm{ml}$ and $\mathrm{Fe}: 85 \div 150 \mu \mathrm{g} / \mathrm{g}$ in blood serum by wet weight is considered normal nowadays.

On the other hand, such traditionally toxic elements as cadmium, arsenic and lead perform important physiological functions when present to a small extent. ${ }^{1}$ Aluminium is one of the metals which are of interest for toxicology. The role of this element in the metabolism of living organisms is still not clear. This problem is very complicated because it is difficult to produce "aluminiumless" diets due to aluminium wide distribution in the environment. In some cases it has been established that an increased content of aluminium in an organism causes various diseases of the lungs and the nervous system. ${ }^{2}$

Among the existing methods atomic fluorescence and atomic absorption methods with flame and flameless atomization are extensively used at present to analyze traces of metals in biological objects.

To our opinion, however, these methods have some essential disadvantages. First, the sample is subjected, as a rule, before analysis to some chemical processes in order to decrease the effect of the matrix on the signal. This may lead to uncontrollable contamination of the sample with the metals contained in the chemicals used. Moreoever, the element being determined may be lost during its chemical extraction. Secondly, in the process of atomization in the inert gas medium a part of the atoms produced is lost as a result of their interaction with the atoms and molecules of the impurities present in the gas. Third, in recording the useful signal no account is taken of the spurious contribution of the annealed matrix molecules to the signal even though their absorption bands can overlap the spectral range used in the analysis. The correction introduced to the final result by the signal from deionized water is not complete, however.

The method of analysis we propose that includes laser stepwise photoionization of atoms combined with thermal atomization of the substance in vacuum is free of all the disadvantages listed above. This new method of analysis is a natural continuation of the series of successful works on laser detection of single atoms (see review papers ${ }^{3,4}$ ). The laser methods of single atom detection and particularly the method of laser stepwise photoionization proposed and realized more than ten years ago ${ }^{5}$ are of great interest for their being used in element trace analysis. It has been repeatedly reported in books and review papers on laser spectroscopy (see, for example Ref. 6). This paper demonstrates how to determine directly by this method 
the trace amounts of elements in biological objects, aluminium in the human blood for example. The analytical procedure consisted in the following: $40 \mathrm{ml}$ of blood in its natural form was introduced into a tantalum cylindrical crucible and dried at 80 to $100^{\circ} \mathrm{C}$ for 3 to 5 minutes. The processes of ashing and atomization of the dry residuum were performed in a vacuum chamber evacuated to a pressure of $10^{-6}$ Torr (Figure 1). During the ashing the crucible temperature was increased in several steps to $1500^{\circ} \mathrm{C}$ for 10 minutes. Atomization was performed at $1800^{\circ} \mathrm{C}$. The evaporating substance was formed into an atomic-molecular beam with the use of diaphragms. In the region between the electrodes the beam was irradiated by two tunable dye lasers with their wavelengths tuned for stepwise excitation of

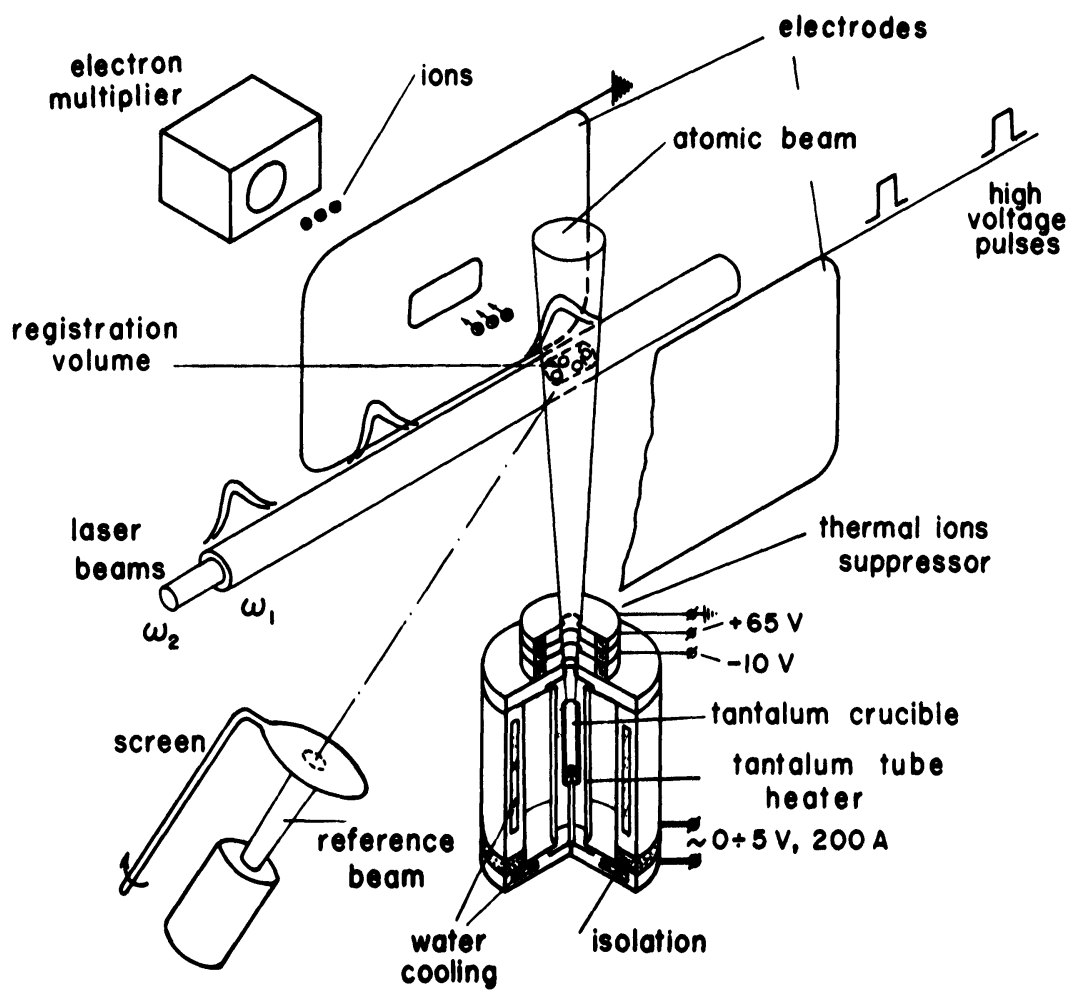

FIGURE 1 Experimental arrangement for analytical photoionization measurements. 
aluminium atoms to a Rydberg state by the following scheme

$$
3^{2} \mathrm{P}_{3 / 2} \stackrel{\lambda_{1}=3961.5 \AA}{\longrightarrow} 4{ }^{2} \mathrm{~S}_{1 / 2} \stackrel{\lambda_{2} \approx 4974 \AA}{\longrightarrow} 15 \mathrm{p}
$$

After the atoms had been excited an electric pulse that ionized all the Rydberg atoms with efficiency approximating unity was fed to the electrodes with a delay of $20 \mathrm{~ns}$. The total efficiency of ionization of aluminium atoms from the $3^{2} \mathrm{P}_{3 / 2}$ state was determined by the efficiency of Rydberg state excitation and it was equal to $10 \%$. The resulting ions were detected with an electron multiplier, then the signal was passed through a boxcar averager to a recorder for signal time scanning. For tuning the radiation of the lasers to the aluminium transitions the vacuum chamber had an additional oven to form a reference beam of aluminium atoms. The experimental setup is described elsewhere in more detail ${ }^{4,7}$.

Figure 2a illustrates a typical time dependence of the ion signal during stepwise heating of the crucible. In the process of the signal recording the wavelength of the first-step laser was periodically tuned off by $\Delta \nu \simeq 3$ to $5 \mathrm{~cm}^{-1}$ from the transition $3 \mathrm{p} \rightarrow 4 \mathrm{~S}$. At such detuning the ionization efficiency of aluminium atoms was reduced by more than two orders of magnitude and we could observe just the background signal from the matrix. The nature of this signal is rather complex. It is probably caused by thermal ionization of the easily ionizable compounds formed at blood matrix decomposition and their photoionization by laser radiation.

For quantitative measurements of the aluminium content in blood an analytical calibration curve was constructed on the basis of aluminium chloride solutions in deionized water (Figure 3 ). The linear part of the curve lies in the aluminium concentration range from $1 \mu \mathrm{g} / \mathrm{ml}$ to $5 \mathrm{ng} / \mathrm{ml}$. The detection limit of the experimental setup for aluminium obtained by extrapolating the analytical curve (dashed line) to the noise level was $10 \mathrm{pg} / \mathrm{ml}$.

Nearly the same detection limit of $\mathrm{Al}$ in pure solutions is possessed by the best commercial analytical instruments, e.g., "Perkin-Elmer", model 5000 with Zeeman background correction. However, their sensitivity worsens almost by two orders comparatively with ours when one makes a direct analysis of blood natural samples or those of serum.

The total aluminium signal for the sample under study was determined by the total "selective" area (the difference between the observ- 


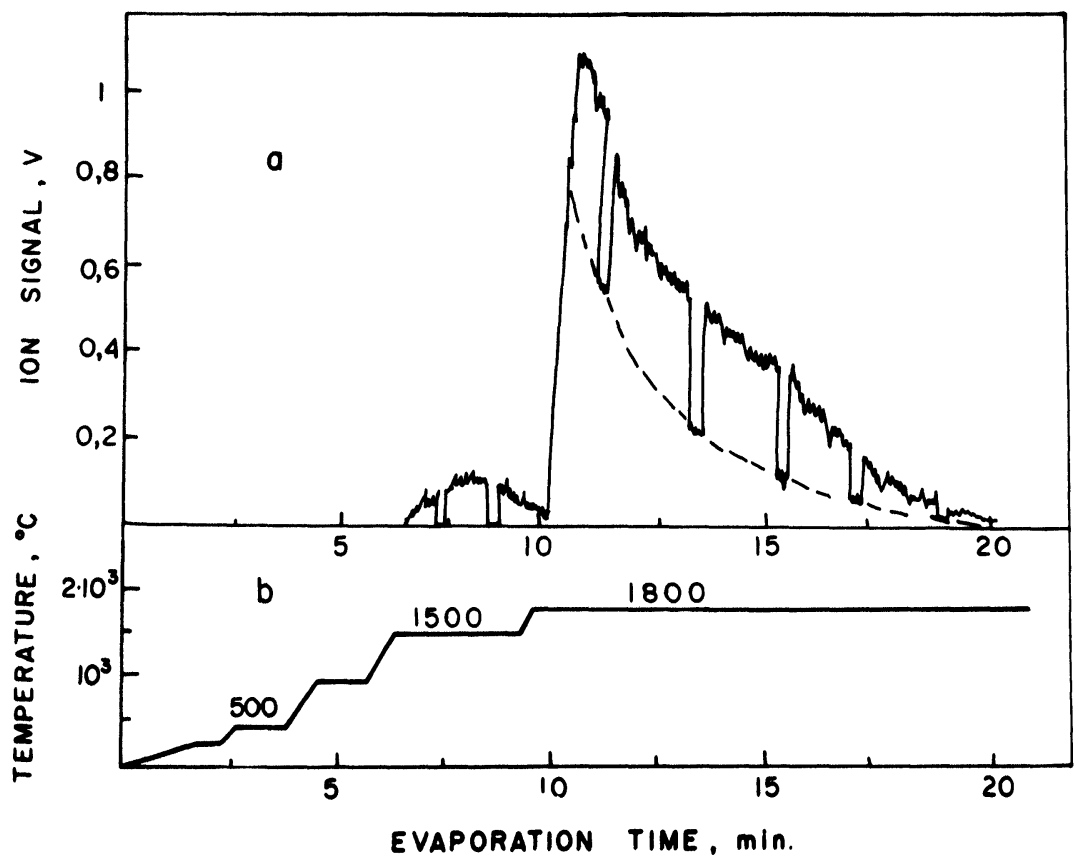

FIGURE 2 (a) Time dependence of the ion signal during stepwise heating of the $40 \mu \mathrm{l}$ blood sample. (b) Time dependence of the crucible temperature.

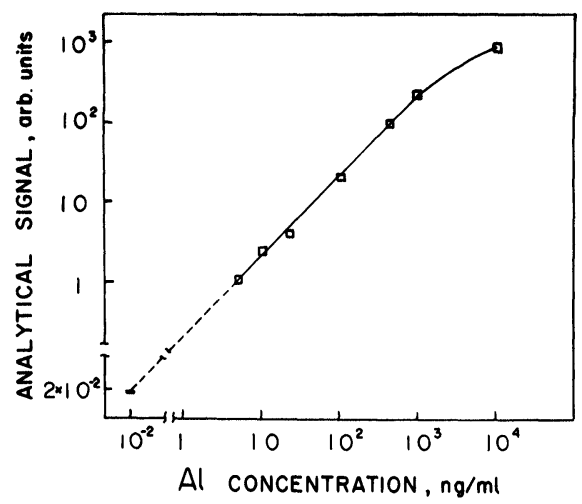

FIGURE 3 Analytical calibration characteristic for $\mathrm{Al}$ on the basis of $\mathrm{AlCl}_{3}$ solutions in deionized water. 
able and background signals) under the signal curve (Figure 2a). The value of aluminium concentration corresponding to such a signal was found from the calibration curve constructed for the aluminium aqueous solutions. The validity of such processing was tested with the additions method. In this case $40 \mu \mathrm{l}$ of blood and $40 \mu \mathrm{l}$ of $\mathrm{AlCl}_{3}$ solution with $100 \mathrm{ng} / \mathrm{ml}$ aluminium content were simultaneously introduced into the crucible. The aluminium signal produced by such a mixture turned out to be additive within the limits of measuring error equal to $10 \%$. This fact proved the absence of the blood matrix influence on the aluminium yield during thermal atomization. The results of measuring the aluminium content in five blood samples lies within $(230 \pm 50) \mathrm{ng} / \mathrm{ml}$.

The experiments carried out show that it is promising to apply the method of laser stepwise photoionization of atoms with thermal atomization of substance in vacuum to analyze elements in biological objects. Modification of the atomizer, improvement of the laser setup parameters, elimination of nonselective ion background make it possible, in principle, to increase the sensitivity of the method by one or two orders.

Some obvious advantages of the proposed technique, such as its universality, the possibility of direct analysis of almost any objects, high sensitivity, elimination of uncontrollable pollutants through atomization in vacuum - enable it to be developed as a new highly sensitive and highly selective method for trace element analysis almost in any matrix.

\section{References}

1. K. Schwarz, in: Clinical Chemistry and Chemical Toxicology, ed. S. S. Brown (Elsevier, Amsterdam, 1977).

2. E. Berman, in: Toxic Metals and their Analysis, ed. L. C. Thomas (Heiden, London, Philadelphia, Rheine, 1979).

3. V. S. Letokhov, in: Chemical and Biochemical Applications of Lasers, Vol. 5, ed. C. B. Moore (Academic Press, New York, London, 1980) pp. 1-38.

4. V. I. Balykin, G. I. Bekov, V. S. Letokhov and V. I. Mishin, Uspekhi Fiz. Nauk (Russian) 132, 293-344 (1980); Sov. Phys.-Uspekhi, 23 (10), 651-678 (1980).

† We were unable to find the results of the direct determination of aluminium content in blood in the related literature. There are only data on aluminium content in serum (see, e.g., Refs. 2 and 8). 
5. V. S. Letokhov, USSR Patent No. 784679 (Appl. 30.03.1970; Published 1.08.1980); R. V. Ambartsumyan, V. P. Kalinin and V. S. Letokhov, Pis'ma ZhETF (Russian) 13, 305 (1971); JETP Lett. 13, 217 (1971).

6. W. S. Letochow, Laserspektroskopie (Vieweg, Braunchweig, 1977); (AkademieVerlag, Berlin 1977) pp. 150-154.

7. G. I. Bekov, E. P. Vidolova-Angelova, V. S. Letokhov and V. I. Mishin, in: Laser Spectroscopy IV, ed. H. Walther and K. W. Rothe, Springer Series in Optical Sciences, Vol. 21 (Springer-Verlag, Berlin, Heidelberg, New York, 1979) pp. 283-294.

8. D. Couri, L. Liss and K. Ebner, Neurotoxicology, 1, 17 (1980). 